\section{L'autophagie et le dernier soupir de la cellule}

Sophie Pattingre, Patrice Codogno
Inserm U756, Université Paris-Sud,

5 , rue Jean-Baptiste Clément, 92296 Châtenay-Malabry, France. sophie.pattingre@u-psud.fr patrice.codogno@u-psud.fr

cela a été récemment discuté dans ces colonnes [10], le «déterminisme » vital ou mortel de l'autophagie, et sa relation avec l'apoptose, sont le fruit pour partie d'acteurs moléculaires communs, notamment par l'interaction entre la protéine anti-apoptotique $\mathrm{Bcl}-2$ et la protéine autophagique Beclin 1 (Atg6) [11]. La modulation de l'autophagie est récemment apparue comme un enjeu thérapeutique dans certaines pathologies comme les maladies neurodégénératives et le cancer [12]. Néanmoins ces nouvelles pistes thérapeutiques nécessitent de mieux maîtriser les acteurs moléculaires qui contrôlent l'autophagie et ses conséquences sur la survie et la mort cellulaire.

\section{Autophagie et phagocytose} des cellules apoptotiques

Qu et al. [13] ont étudié le rôle de gènes - connus pour leur rôle dans la formation de l'autophagosome (Atg5 et Beclin 1) - dans la formation des corps embryoïdes et la cavitation dans un modèle in vitro qui reproduit les premières étapes de l'embryogenèse. En effet, les cellules souches embryonnaires (cellules ES) murines, cultivées en l'absence du facteur LIF (leukemia inhibiting factor) s'engagent spontanément dans un processus de différenciation. Après une première étape où les cellules s'agrègent, on observe la formation d'un corps embryoïde comprenant au centre des cellules ectodermiques et en périphérie des cellules de nature endodermique. À partir du $6^{\mathrm{e}}$ jour, cet agrégat se creuse, les cellules ectodermiques internes mourant par apoptose. Cette cavitation, dont la taille augmente jusqu'au 
$2^{\mathrm{e}}$ jour de culture, mime la formation de la cavité amniotique (Figure 1).

Mais, si cette expérience est faite avec des cellules ES Atg5 $5^{-/-}$ou Beclin 1 $^{-/-}$ (cellules $\varepsilon S$ déficientes en autophagie = $\varepsilon^{a}{ }^{a t-}$ ), la cavitation ne se produit pas.
Deux hypothèses ont alors été émises: (1) les cellules ne font plus d'apoptose ; ou (2) les cellules apoptotiques ne sont pas éliminées. En effet, in vivo, l'étape ultime de l'apoptose est l'élimination de la cellule apoptotique par des cel-

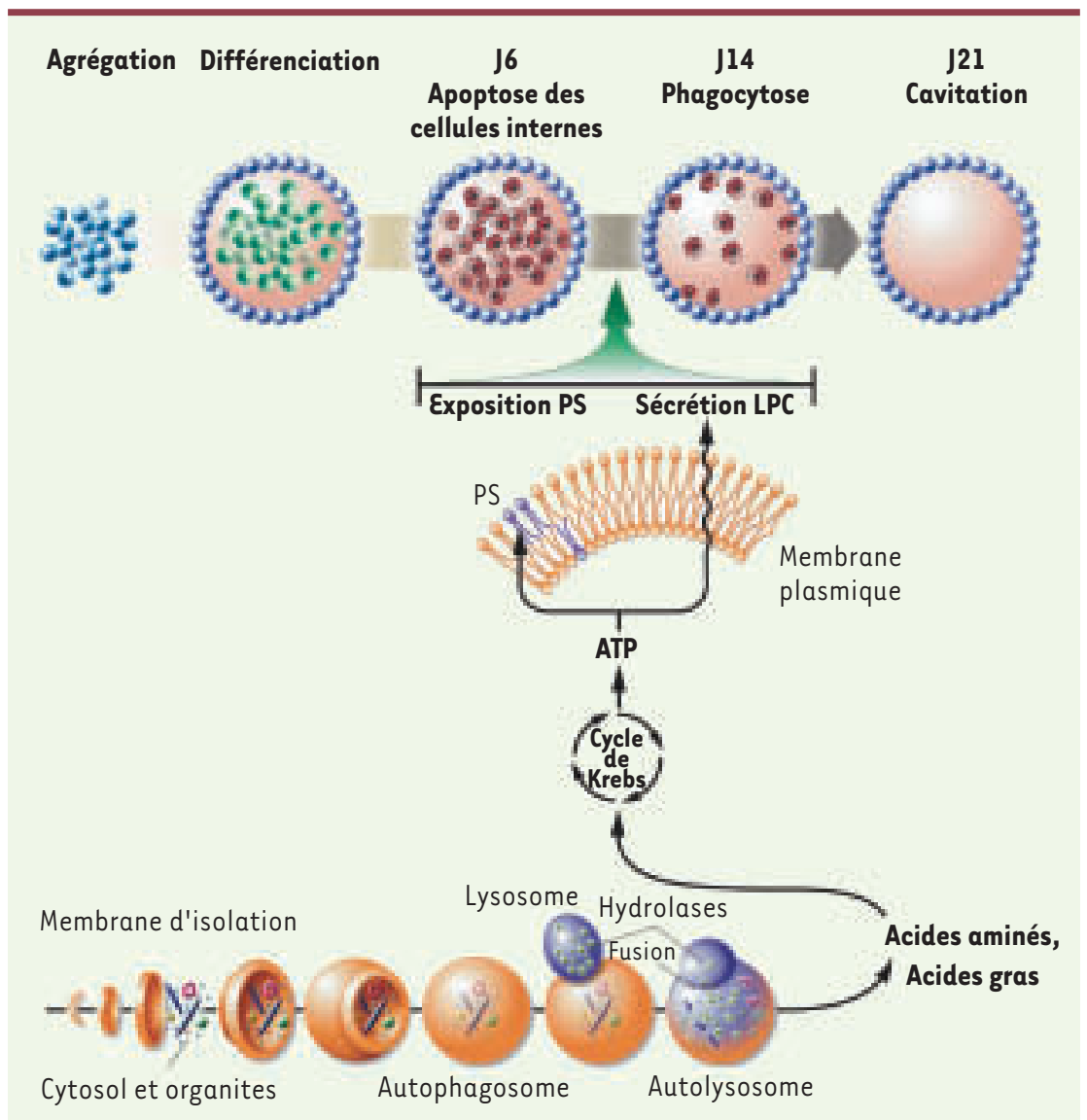

Figure 1. L'autophagie permet l'élimination des cellules apoptotiques. L'autophagie débute à partir d'une membrane d'isolation qui forme une vacuole ou autophagosome séquestrant des portions de cytoplasme. Le contenu intravacuolaire sera dégradé par les enzymes lysosomales après fusion de l'autophagosome et du lysosome. La dégradation est dans de nombreux cas un mécanisme cytoprotecteur soit par l'élimination des molécules ou structures pouvant mettre en péril la viabilité cellulaire soit par la libération d'acides aminés et d'acides gras indispensables au métabolisme et à la production d'ATP par le cycle de Krebs notamment en période de jeûne. L'étude de Qu et al. [13] montre que la production d'ATP via l'autophagie est nécessaire à l'expression de signaux émis par la cellule apoptotique pour sa phagocytose par les cellules voisines. Deux types de signaux sont émis par la cellules apoptotique : la translocation de la phosphatidylsérine (PS) du feuillet interne vers le feuillet externe de la membrane plasmique (eat-me signal, signal de phagocytose) et la sécrétion de la lysophosphatidylcholine (LPC) (come-and-get me signal, signal chimioattractant). Ce rôle de l'autophagie a été démontré dans les corps embryoïdes qui reproduisent les premières étapes de l'embryogenèse. Les cellules ectodermiques (vert) déclenchent une mort programmée apoptotique. L'élimination des cellules ectodermiques apoptotiques (rouge) par les cellules endodermiques (bleu) de la couche externe permet la cavitation. L'inhibition de l'autophagie se traduit par un engorgement de la cavité par des cellules ectodermiques apoptotiques qui n'ont pas émis les signaux PS et LPC nécessaires à leur élimination. lules phagocytaires (professionnelles ou non). Dans le modèle étudié, les cellules ectodermiques apoptotiques sont normalement phagocytées par les cellules endodermiques, mais ce n'est pas le cas dans les corps embryoïdes aut-

Le mécanisme par lequel l'autophagie permet la phagocytose des cellules apoptotiques a également été élucidé par l'étude de Qu et al. [13]. En effet, deux types de signaux sont émis par la cellule apoptotique [14]: (1) un signal de reconnaissance appelé eatme signal qui correspond à la translocation de la phosphatidylsérine du feuillet interne vers le feuillet externe de la membrane plasmique; (2) un signal chimioattractant pour les cellules phagocytaires appelé come-andget me signal. Ce signal, quant à lui, repose sur la synthèse et la sécrétion de la lysophosphatidylcholine par la cellule apoptotique.

\section{Comment l'autophagie permet-elle l'élimination des cellules apoptotiques?}

L'une des fonctions importantes de l'autophagie, qui s'exerce lors de périodes de jeûne [6], est de maintenir le niveau d'ATP cellulaire. Or, les auteurs ont observé une diminution du niveau d'ATP dans les corps embryoïdes ${ }^{\text {aut- }}$ et la supplémentation des cellules ES $^{\text {aut- }}$ par du méthylpyruvate (substrat du cycle de Krebs) restaure le niveau d'ATP et la cavitation (Figure 1). À partir de ces résultats, les auteurs suggèrent que l'autophagie est essentielle pour fournir l'énergie nécessaire à l'émission des signaux requis pour la phagocytose des cellules apoptotiques.

Ces résultats ont enfin été validés in vivo dans la rétine et le poumon au cours du développement des souris $\operatorname{Atg}^{-/-}$. Ce défaut d'élimination des cellules apoptotiques conduit en effet à l'accumulation de macrophages, suggérant la présence d'un foyer inflammatoire provoqué par la libération du contenu des corps apoptotiques dans l'espace extracellulaire (nécrose secondaire). 


\section{En conclusion}

Ce travail démontre un rôle inattendu pour l'autophagie à l'étape ultime du programme apoptotique en permettant l'élimination par phagocytose de la cellule morte.

Comme tout travail novateur, cette étude pose un certain nombre de questions, et nous en relèverons deux :

- Alors que des nutriments sont présents dans l'environnement cellulaire, pourquoi l'autophagie est-elle nécessaire à la production d'ATP permettant la mise en place des signaux de phagocytose dans les corps embryoïdes?

- Pourquoi les phénotypes des souris Atg5 $5^{-/-}$et Beclin $1^{-/-}$sont-ils différent? $\varepsilon n$ effet, aucune anomalie majeure n'est observée au cours du développement des souris $A \operatorname{tg} 5^{-/-}$[6] alors que celui des souris Beclin $1^{-/-}$s'arrête au jour $\mathrm{J} 7,5$ de gestation $[15,16]$ ? Deux réponses non exclusives peuvent rendre compte de cette différence: soit la protéine Beclin 1 est impliquée dans d'autres processus que l'autophagie, soit il existe un phénomène de compensation à l'invalidation du gène $\operatorname{Atg} 5 . \diamond$

Autophagy and clearence

of apoptotic cells

\section{RéFÉRENCES}

1. De Duve C, Wattiaux R. Functions of lysosomes. Annu Rev Physiol 1966; $28: 435$.

2. Codogno $P$. Les gènes atg et la macroautophagie. Med Sci (Paris) 2004 ; $20: 734-6$.

3. Metzstein MM, Stanfield GM, Horvitz HR. Genetics of programmed cell death in C. elegans: past, present and future. Trends Genet $1998 ; 14: 410-6$.

4. Hara T, Nakamura K, Matsui M, et al. Suppression of basal autophagy in neural cells causes neurodegenerative disease in mice. Nature 2006; $441: 885-9$.

5. Komatsu M, Waguri S, Chiba T, et al. Loss of autophagy in the central nervous system causes neurodegeneration in mice. Nature 2006; 44 : 880-4.

6. Kuma A, Hatano M, Matsui $M$, et al. The role of autophagy during the early neonatal starvation period. Nature 2004 ; 432 : 1032-6.
7. Boya P, Gonzalez-Polo RA, Casares N, et al. Inhibition of macroautophagy triggers apoptosis. Mol Cell Biol $2005 ; 25: 1025-40$.

8. Deretic V. Autophagy as an immune defense mechanism. Curr Opin Immunol 2006 ; 18: 375-82.

9. Gozuacik D, Kimchi A. Autophagy and cell death. Curr Top Dev Biol 2007 ; 78 : 217-45.

10. Espert L, Denizot M, Grimaldi M, et al. Autophagie et destruction des lymphocytes T CD4 par le VIH-1. Med Sci (Paris) $2006 ; 22: 677-8$.

11. Pattingre S, Tassa A, Qu X, et al. Bcl-2 antiapoptotic proteins inhibit Beclin 1-dependent autophagy. Cell 2005 ; 122 : 927-39.

12. Rubinsztein DC, Gestwicki JE, Murphy LO, Klionsky DJ. Potential therapeutic applications of autophagy. Nat Rev Drug Discov 2007 ; 6 : 304-12.

13. Qu X, Zou Z, Sun Q, et al. Autophagy gene-dependent clearance of apoptotic cells during embryonic development. Cell. 2007 ; 128 : 931-46.

14. Grimsley C, Ravichandran KS. Cues for apoptotic cell engulfment: eat-me, don't eat-me and come-get-me signals. Trends Cell Biol $2003 ; 13: 648-56$.

15. Qu X, Yu J, Bhagat $G$, et al. Promotion of tumorigenesis by heterozygous disruption of the beclin 1 autophagy gene. J Clin Invest 2003 ; 112 : 1809-20.

16. Yue Z, Jin S, Yang C, et al. Beclin 1 , an autophagy gene essential for early embryonic development, is a haploinsufficient tumor suppressor. Proc Natl Acad Sci USA 2003 ; 25 : 15077-82.

\section{NOUVELLE}

\section{Le danio zébré révèle l'odyssée des précurseurs hématopoiiétiques au cours du développement des embryons de vertébrés}

Karima Kissa, Emi Murayama, Philippe Herbomel
Unité Macrophages et Développement de l'Immunité, CNRS-URA 2578, Institut Pasteur, 25, rue du Docteur Roux, 75724 Paris Cedex 15, France. herbomel@pasteur.fr
> Les cellules souches hématopoïétiques (CSH) sont à l'origine des divers types de cellules sanguines et de leur production continuelle chez les vertébrés adultes. Au cours du développement des mammifères, la genèse des cellules du sang se fait en deux vagues successives $[1,2]$. La première vague, dite primitive, est transitoire, et produit dans le sac vitellin les érythrocytes et les macrophages primitifs de l'embryon. Puis une seconde vague, dite définitive, produit, à partir de CSH, l'ensemble des cellules sanguines caractérisées chez l'adulte, en particulier la diversité des lymphocytes. Cette hématopoï̀se définitive a d'abord lieu dans le foie foetal, puis dans la moelle osseuse, et parallèlement dans le thymus pour ce qui est de la maturation des lymphocytes T. Au cours de l'embryogenèse, le premier site où des cellules présentant le potentiel de CSH ont été identifiées est une région intra-embryonnaire appelée AGM (aorte-gonade-mésonephros) (voir Nouvelle d'Isabelle Godin et Ana Cumano, page 681 de ce numéro) [3]. Ce potentiel de CSH a été mis en évidence en montrant la capacité qu'ont ces cellules, lorsqu'elles sont injectées dans une souris adulte irradiée, de restaurer durablement toute I'hématopoïèse.

De là, il a été conclu que les organes hématopoïétiques transitoires (foie fœtal) puis définitifs (thymus, moelle osseuse) sont successivement colonisés par des CSH initialement nées dans I'AGM. Bien que ce principe soit admis, aucune démonstration directe d'une continuité de lignage cellulaire depuis l'AGM jusqu'aux organes hématopoïétiques définitifs n'a jamais été faite (voir Nouvelle d'Isabelle Godin et Ana Cumano, page 681 de ce numéro) [3].

Malgré les divergences apparues entre les mammifères et les poissons au cours de l'évolution, le patron de signalisation moléculaire gouvernant l'hématopoïèse a été très conservé [4]. Aussi, parce que l'embryon de danio zébré (zebrafish, d'où l'anglicisme poisson zèbre) se développe 